\section{Current treatment options in elderly patients with chronic lymphocytic leukemia}

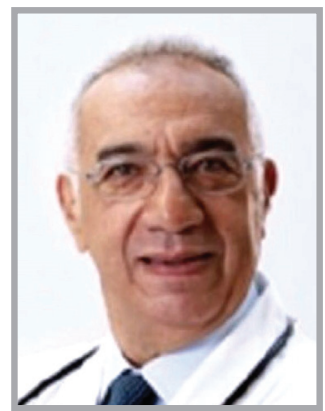

Stefano Molica*

\author{
“Despite differences in the overall \\ survival between younger and older \\ chronic lymphocytic leukemia patients, \\ clinicobiologic characteristics at \\ diagnosis are quite similar. What is \\ different is the number of \\ comorbidities."
}

International Journal of Hematologic Oncology
First draft submitted: 28 June 2016; Accepted for publication: 11 July 2016; Published online: 23 September 2016

In contrast to the general belief, the majority of elderly chronic lymphocytic leukemia (CLL) patients die of the disease. In a large, monoinstitutional series (i.e., the Mayo Clinic database), age at diagnosis was demonstrated to be a strong independent prognostic factor not only for the general population of CLL patients but also for Rai stage 0 patients [1]. It is not surprising, therefore, that age has been included in several prognostic models $[2,3]$.

Despite differences in the overall survival (OS) between younger and older CLL patients, clinicobiologic characteristics at diagnosis are quite similar. What is different is the number of comorbidities [4]. Older patients tend to have a higher number of comorbidities, which can in some instances be rather severe. As a result, tolerability to treatment is poor and several patients discontinue therapy because of the occurrence of severe toxicities [4].

When we review the different options that are available for patients who do not have a deletion of chromosome $17 \mathrm{p}$ and/or TP53 mutations, there are several possibilities. The combination of chemoimmunotherapy fludarabine, cyclophosphamide and rituximab or bendamustine and rituximab is an option for patients who are fitter, younger and have preserved renal function. This type of combination induces long-lasting remissions in the majority of patients (i.e., remission rates in $90 \%$ of patients) $[5,6]$. These results, however, do not apply to patients who are on the older side, with comorbidities and are more frail. In the CLL-11 trial, which randomized patients with multiple comorbidities and a median age of 73 years to receive chlorambucil, chlorambucil plus rituximab or chlorambucil plus obizutunumab, the investigators of the German CLL Study Group found that the combination including obizutunumab significantly increased OS and progression-free survival (PFS) [7]. Unlike the CLL-11 study, the design of the COMPLEMENT- 1 trial lacks a comparison to rituximab [8]. In the COMPLEMENT-1 trial, CLL patients considered fludarabine-ineligible were randomized to receive ofatumumab plus chlorambucil versus chlorambucil monotherapy. PFS, the primary end point of the
*Department of Hematology-Oncology, Azienda Ospedaliera Pugliese-Ciaccio, Viale Pio X, 88100 Catanzaro, Italy; Tel.: +39 096188 3001; Fax: +39 096188 3467; smolica@libero.it

\section{KEYWORDS}

- chronic lymphocytic leukemia

- CLL • comorbidities • elderly

- treatment

\section{"Many unresolved issues remain with regard to the first-line treatment of elderly patients with chronic lymphocytic leukemia.”}




\section{"Chemoimmunotherapy has the benefit of limited duration of therapy with long remission, in contrast to the targeted agents that, to date, have largely been studied as continuous single agents."}

study, was significantly improved with the association of ofatumumab plus chlorambucil [8]. Although the CLL-11 and COMPLEMENT-1 trials were different with respect to criteria of patient selection and doses of chlorambucil utilized, they have established an important breakthrough in the treatment of elderly/unfit CLL patients.

The current CLL treatment paradigm is now evolving as a consequence of the understanding of the disease's pathophysiology. The B-cell receptor regulates fundamental proliferation and survival mechanisms for malignant B cells [9]. These functions are mediated by signals that are transmitted intracellularly downstream through several kinases, including Lyn kinase, spleen tyrosine kinase, PI3K, BTK and others. Targeting these kinases, in particular the BTK and the PI3K signaling pathways, has shown remarkable clinical activity in patients with CLL and with other B-cell malignancies [9].

From a clinical standpoint, a Phase III study of ibrutinib versus ofatumumab, which enrolled patients with relapsed or refractory CLL (RESONATE), demonstrated that singleagent ibrutinib was superior to ofatumumab, with a risk of progression that was $78 \%$ lower and a risk of death that was $57 \%$ lower [10]. In 31 previously untreated patients with CLL who were 65 years of age or older, the estimated rate of PFS at 30 months was $96 \%$, and the OR rate was $97 \%$. These findings qualify ibrutinib single agent as a potential upfront treatment in the elderly with CLL [11].

In an older population of previously untreated patients with CLL, many of whom had coexisting conditions, oral ibrutinib was administered continuously in the context of a multicenter open-label, randomized Phase III trial (RESONATE-2; study number, PCYC-1115-CA) in comparison to chlorambucil. Treatment with ibrutinib resulted in significantly longer PFS (i.e., $89.9 \%$ at 18 months vs $51.5 \%$ of the chlorambucil arm) [12]. Ibrutinib also significantly prolonged OS with an 18-month survival rate of $97.8 \%$, compared with $87.2 \%$ in the chlorambucil arm. The safety of ibrutinib in this patient population was consistent with previously reported studies. Overall, adverse events leading to treatment discontinuation were less frequent with ibrutinib than with chlorambucil ( 9 vs $23 \%$, respectively). The most common adverse events of any grade were diarrhea $(42 \%)$, fatigue $(30 \%)$, cough $(22 \%)$ and nausea (22\%). Hypertension occurred at a higher rate in the ibrutinib arm (14\%; grade 3 in 4). Four patients treated with ibrutinib experienced grade 3 hemorrhage and one experienced grade 4 hemorrhage [12].

Many unresolved issues remain with regard to the first-line treatment of elderly patients with CLL. Chemoimmunotherapy, generally based on a combination of chlorambucil and an antiCD20 monoclonal antibody, remains an appropriate option for many elderly patients with CLL and in particular those with the most favorable features in the front-line setting. The use of targeted novel agents has changed the therapeutic landscape for treating relapsed, refractory CLL. Chemoimmunotherapy has the benefit of limited duration of therapy with long remission, in contrast to the targeted agents that, to date, have largely been studied as continuous single agents. Several questions remain for further investigation, including those assessing the optimal length of therapy, the sequencing of agents and understanding responses with class switching as well as questions focusing on the mechanisms of overcoming resistance [13].

\section{Financial \& competing interests disclosure}

The author has no relevant affliations or financial involvement with any organization or entity with a financial interest in or financial conflict with the subject matter or materials discussed in the manuscript. This includes employment, consultancies, honoraria, stock ownership or options, expert testimony, grants or patents received or pending, or royalties.

No writing assistance was utilized in the production of this manuscript.

\section{References}

1 Shanafelt TD, Rabe KG, Kay NE et al. Age at diagnosis and the utility of prognostic testing in patients with chronic lymphocytic leukemia. Cancer 116, 4777-4787 (2010). Prognostic nomogram and index for overall

survival in previously untreated patients with chronic lymphocytic leukemia. Blood 109(11), 4679-4685 (2007)

3

The International CLL-IPI Working Group. An international prognostic index for patients with chronic lymphocytic leukaemia (CLL-IPI): a meta-analysis of individual patient data. Lancet Oncol. 7, 779-790 (2016).

4 Goede V, Cramer P, Busch R et al. Interactions between comorbidity and treatment of chronic lymphocytic leukemia: results of German Chronic Lymphocytic Leukemia Study Group trials. Haematologica 99(6), 1095-1100 (2014). 
5 Hallek M, Fischer K, Fingerle-Rowson G et al. Addition of rituximab to fludarabine and cyclophosphamide in patients with chronic lymphocytic leukaemia: a randomised, open-label, Phase 3 trial. Lancet 376, 1164-1174 (2010).

6 Eichhorst B, Fink AM, Bahlo J et al. First-line chemoimmunotherapy with bendamustine and rituximab versus fludarabine, cyclophosphamide, and rituximab in patients with advanced chronic lymphocytic leukaemia (CLL10): an international, open-label, randomised, Phase 3, noninferiority trial. Lancet Oncol. doi: 10.1016/ S1470-2045(16)30051-30051 (2016) (Epub ahead of print).

7 Goede V, Fischer K, Busch R et al. Obinutuzumab plus chlorambucil in patients with CLL and coexisting conditions. N. Engl. J. Med. 370 (12), 1101-1110 (2014).

8 Hillmen P, Robak T, Janssens A et al. Chlorambucil plus ofatumumab versus chlorambucil alone in previously untreated patients with chronic lymphocytic leukaemia (COMPLEMENT 1): a randomised, multicentre, open-label Phase 3 trial. Lancet 385(9980), 1873-1883 (2015).

9 Cramer P, Hallek M, Eichhorst B. State-ofthe-art treatment and novel agents in chronic lymphocytic leukemia. Oncol. Res. Treat. 39(1), 25-32 (2016).

10 Byrd JC, Brown JR, O’Brien S et al. Ibrutinib versus ofatumumab in previously treated chronic lymphoid leukemia. N. Engl. J. Med. 371, 213-223 (2014).
11 Byrd JC, Furman RR, Coutre SE et al. Three-year follow-up of treatment-naive and previously treated patients with CLL and SLL receiving single-agent ibrutinib. Blood 125 , 2497-2506 (2015).

12 Burger JA, Tedeschi A, Barr PM et al. Ibrutinib as initial therapy for patients with chronic lymphocytic leukemia. N. Engl. J. Med. 373(25), 2425-2437 (2015).

13 Brown JR, Hallek MJ, Pagel JM. Chemoimmunotherapy versus targeted treatment in chronic lymphocytic leukemia: when, how long, how much, and in which combination? Am. Soc. Clin. Oncol. Educ. Book 35, e387-e398 (2016). 